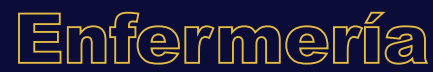

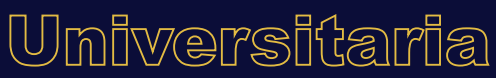

\section{Relación entre el nivel de ansiedad y rendimiento académico en estudiantes de enfermería}

\author{
Relationship between anxiety level and \\ academic performance among nursing students
}

\section{Relação entre o nível de ansiedade e desempenho acadêmico em estudantes de enfermagem}

\section{Mosqueira-Soto ${ }^{a^{*}}$, M. Poblete-Troncoso ${ }^{b_{2}}$}

ORCID

a $\underline{0000-0002-6845-7061}$

b $0000-0003-4068-1253$

${ }^{1}$ Universidad Católica del Maule, Facultad de Ciencias de la Salud, Departamento de Enfermería, Talca, Chile

${ }^{2}$ Universidad Católica del Maule, Facultad de Ciencias de la Salud, Centro de Investigación del Cuidado, Departamento de Enfermería, Talca, Chile

Recibido: 27 enero 2020

Aceptado: 5 septiembre 2020

\section{RESUMEN}

Introducción: La ansiedad ante exámenes es un tipo de ansiedad de ejecución y puede afectar el rendimiento académico, en especial en los estudiantes de enfermería que sufren altos niveles de estrés.

Objetivo: Establecer la relación entre el nivel de ansiedad, el rendimiento académico y variables sociodemográficas en estudiantes de enfermería de la Universidad Católica del Maule.

Metodología: Estudio cuantitativo, descriptivo y de corte transversal. La muestra fue censal, de 94 estudiantes. Para la recolección de los datos se utilizaron dos instrumentos:

*Autora para correspondencia. Correo electrónico: cmosqueira@ucm.cl https://doi.org/10.22201/eneo.23958421e.2020.4.1053

1665-7063/@ 2020 Universidad Nacional Autónoma de México, Escuela Nacional de Enfermería y Obstetricia. Este es un artículo Open Access bajo la licencia CC BY-NC-ND (http://creativecommons.org/licenses/by-nc-nd/4.o/). 
de antecedentes sociodemográficos (edad, género, colegio de procedencia, lugar de origen y ocupación del estudiante); y el Cuestionario de Ansiedad frente a los Exámenes (CAEX). El rendimiento académico se obtuvo del Examen Clínico Objetivo Estructurado (OSCE por sus siglas en inglés). Para el análisis de los datos se usó T-Student y Anova.

Resultados: Se observó una relación significativa entre el rendimiento académico y el semestre que cursaban los estudiantes. Los estudiantes de los semestres más avanzados tuvieron mejor rendimiento $(\mathrm{p}=0.015)$. El alumno que trabaja y estudia presentó mejor rendimiento en el examen OSCE con respecto al que sólo estudia $(p=0.027)$. El sexo femenino presentó diferencias significativas con el nivel ansiedad ( $p=0.038$ ).

Conclusión: Los niveles de ansiedad se encontraron entre los rangos bajos y medios, se sugiere mantenerlos para promover aprendizajes significativos. Resulta necesario analizar la planificación curricular para reestructurar las mallas curriculares y así apoyar a los estudiantes trabajadores al hacer compatible el trabajo con el estudio, con la finalidad de mejorar su calidad de vida.

Palabras clave: Ansiedad; educación en enfermería; rendimiento académico; Chile.

ABSTRACT

Introduction: Anxiety towards tests can impact academic performance, especially among nursing students with high levels of stress.

Objective: To confirm the relationship between the level of anxiety, the academic performance, and some social and demographic variables among nursing students in the Universidad Católica del Maule.

Methodology: This is a quantitative, descriptive, and transversal study. The sample was constituted by 94 students. Data were collected through 3 instruments: (a) a social and demographic data questionnaire (age, gender, school of precedence, place of origin, and occupation); (b) a Questionnaire of Anxiety Towards Exams (QAEX); c) the Objective School Clinical Exam (OSCE). Student's t and ANOVA were calculated.

Results: A significant relationship was observed between academic performance and the school semester, with students in their last semesters (senior) demonstrating better performances $(p=0.015)$. Students working and studying showed better results in the OSCE in comparison to those only studying $(p=0.027)$. Female students showed significant differences in terms of their levels of anxiety $(p=0.038)$.

Conclusion: Anxiety was found to be in the low and middle levels. From the findings, it is suggested to avoid high levels of anxiety among students and to adjust, when necessary, the curriculum in order to support students who work and study at the same time.

Keywords: Anxiety; education, nursing; academic performance; Chile.

\section{RESUMO}

Introdução: A ansiedade causada pelos testes é um tipo de ansiedade de execução e pode afetar o desempenho acadêmico, em especial nos estudantes de enfermagem que sofrem altos níveis de estresse.

Objetivo: Estabelecer a relação entre o nível de ansiedade, o desempenho acadêmico e variáveis sociodemográficas em estudantes de enfermagem da "Universidad Católica del Maule". 
Metodologia: Estudo quantitativo, descritivo e de corte transversal. A amostra foi censo, de 94 estudantes. Para a coleta dos dados utilizaram-se dois instrumentos: de antecedentes sociodemográficos (idade, género, escola de procedência, local de origem e ocupação do estudante); e o Questionário de Ansiedade causada pelos testes (CAEX). O desempenho acadêmico foi obtido do Exame Clínico Objetivo Estruturado (OSCE por suas siglas em inglês). Para a análise dos dados foram usados T-Student e Anova.

Resultados: Observou-se uma relação significativa entre o desempenho acadêmico e o semestre que cursavam os estudantes. Os estudantes dos semestres mais avançados tiveram melhor desempenho ( $p=0.015)$. O aluno que trabalha e estuda apresentou melhor desempenho no exame OSCE em relação ao aluno que apenas estuda $(\mathrm{p}=0.027)$. O sexo feminino apresentou diferenças significativas com o nível de ansiedade $(p=0.038)$.

Conclusão: Os níveis de ansiedade encontraram-se entre as faixas baixas e médias, sugerese mantê-los para promover aprendizagens significativas. Resulta necessário analisar o planejamento curricular para reestruturar as redes curriculares e assim apoiar os estudantes trabalhadores e fazer compativel o trabalho com o estudo, com a finalidade de melhorar sua qualidade de vida.

Palavras chave: Ansiedade; educação em enfermagem; desempenho acadêmico; Chile.

\section{INTRODUCCIÓN}

El aprendizaje, como parte de la educación universitaria en el área de la salud y en enfermería, se concibe como un proceso continuo de adquisición de hábitos inherentes a las competencias profesionales. Para que el aprendizaje sea efectivo deben contemplarse al menos tres áreas específicas: cognitiva, afectiva y psicomotora ${ }^{1,2}$. Estas áreas requieren cimentarse en la formación de los estudiantes para otorgar cuidados de calidad a las personas que tienen diversos problemas de salud.

La educación en enfermería incluye en el desarrollo de los aprendizajes una formación que no sólo contempla la incorporación de conocimientos, sino también precisa de la integración de habilidades, actitudes y valores, necesarios para formar profesionales competentes en el área. Asimismo, este proceso educativo debe estar alineado con el contexto, los rápidos cambios socioculturales y las necesidades emergentes de la sociedad actual.

De acuerdo con Benner³, es importante el saber práctico en la formación de los estudiantes de enfermería, pues refiere lo importante de las inteligencias múltiples para el conocimiento humano. La mayoría de los individuos tienen la totalidad de estas inteligencias, cada una desarrollada de un modo y a un nivel particular, producto de la dotación biológica de cada persona, de su interacción con el ambiente y de la cultura en que crecen, las cuales se combinan y utilizan de manera personal y única.

Derivado de lo anterior, es posible señalar que la formación en enfermería no sólo demanda un proceso educativo adecuado o apropiado para el desarrollo de las competencias en el área, sino que además requiere desarrollar estrategias que permitan una evaluación integral de las competencias que están siendo adquiridas. Sin embargo, tradicionalmente, la evaluación de las competencias se ha basado en la medición de conocimientos mediante la aplicación de diversos tipos de exámenes4 que no siempre cumplen con los criterios de objetividad, validez y confiabilidad que se necesitan. De igual forma, el escenario actual ha llevado a la necesidad imperiosa de desarrollar nuevos métodos 
de educación y evaluación destinados a calificar competencias clínicas profesionales. Ahora bien, éstos métodos no han sido evaluados y muchos de ellos pueden ocasionar estrés y ansiedad en los estudiantes.

Gutiérrez Calvo y Avero señalan que la alta ansiedad ante exámenes reduce la eficiencia en el aprendizaje, dado que disminuye la atención, la concentración y la capacidad de retención, lo que conlleva al deterioro en el rendimiento académico5. La ansiedad puede generar efectos adversos en el desempeño académico, así como en la autoestima, las relaciones con los compañeros, el estado de ánimo y la salud mental del estudiante afectado, lo que pudiera desencadenar el fracaso académico ${ }^{6}$

La ansiedad que ocurre frente a los exámenes es reconocida como un tipo de ansiedad de ejecución ${ }^{7}$ y se refiere a lo que puede experimentar una persona en una situación donde importa mucho el rendimiento o la correcta ejecución de una tarea, o cuando existe una gran presión por hacer las cosas bien. Por otro lado, el rendimiento académico es una medida de las capacidades respondientes o indicativas que manifiesta, en forma estimativa, lo que una persona ha aprendido como consecuencia de un proceso de instrucción o formación ${ }^{8}$. Cabe destacar que en la mayor parte de las instituciones educativas el rendimiento académico pasa a ser un indicador cuantitativo de la nota o una categoría que evalúa si se aprueba o no la asignatura o el semestre.

Las consecuencias que provoca la ansiedad sobre el rendimiento académico se han documentado ampliamente en la literatura científica, puesto que los elevados niveles de ansiedad ante los exámenes ejercen un efecto negativo sobre éste y conllevan un alto riesgo de deserción universitaria. Como se ha señalado, la ansiedad que afrontan los estudiantes universitarios durante su formación académica puede constituir un grave problema, ya que al malestar psíquico se le suma la dificultad para obtener un buen rendimiento académico al verse sobrepasada su capacidad de resiliencia, situación que llega a poner en riesgo su salud ${ }^{9,10}$.

Es común encontrarnos con estudiantes que presentan manifestaciones de ansiedad cuando se ven sometidos a diversos procesos de evaluación, síntomas que pueden ser desde expresiones autodesvalorativas hasta fisiológicas como sudoración copiosa y/o enrojecimiento delas mejillas, o manifestaciones conductuales como la inquietud ${ }^{11,12}$. A este fenómeno se le ha denominado ansiedad de evaluación, concepto que engloba situaciones percibidas como amenazantes por una persona, las cuales repercuten tanto a nivel cognitivo como a nivel fisiológico y conductual7.

Derivado de lo anterior y considerando que el rendimiento de los estudiantes puede verse afectado por la ansiedad, este estudio tuvo como objetivo establecer la relación entre el nivel de ansiedad, el rendimiento académico en el Examen Clínico Objetivo Estructurado (OSCE por sus siglas en inglés) y las variables sociodemográficas de estudiantes del tercer, quinto y séptimo semestre académico de la carrera de enfermería de una universidad chilena, con el propósito de establecer estrategias de manejo de la ansiedad, mejorar el rendimiento académico y promover aprendizajes significativos en los alumnos. A su vez, la pregunta de investigación fue: ¿existe relación entre el nivel de ansiedad, rendimiento académico y las variables sociodemográficas de los estudiantes?

\section{METODOLOGÍA}

Estudio cuantitativo, descriptivo y de corte transversal desarrollado en el año 2015, en estudiantes de enfermería de la Universidad Católica del Maule, Chile.

La población de alumnos de enfermería por semestre eran 400, de ellos se tomó una muestra censal constituida por 94 estudiantes que pertenecían al tercer, quinto y séptimo semestre de 
la carrera de enfermería y cuya participación fue voluntaria. Los criterios de inclusión de la muestra correspondieron a los estudiantes que cumplieron con los requisitos de aprobación de los módulos y actividades curriculares teórico prácticas y que se presentaron a rendir el OSCE, por tanto, se consideró como exclusión, estudiantes que habían reprobado la teoría o práctica de los módulos.

La recolección de datos se llevó a cabo en el mes de diciembre de 2015, previo acuerdo con las coordinadoras de los módulos dónde se realizó el examen evaluativo OSCE. El momento de la recolección de éstos fue después de hacer el examen, en la sala de post-espera. Para la obtención de los datos, el investigador elaboró un instrumento que constó de dos apartados. Mientras que el primero contenía preguntas sobre datos sociodemográficos relacionados con la edad, el sexo, semestre académico, procedencia, ocupación y establecimiento de procedencia; el segundo utilizó el CAEX (Cuestionario de Ansiedad ante los exámenes), instrumento ${ }^{13,6}$ diseñado por Luis Valero Aguayo en 1999, académico de la Facultad de Psicología, Málaga, España, para evaluar la presencia de ansiedad.

Cabe destacar que para el presente estudio, el CAEXfue adaptado en sus propiedades semánticasy piloteado con una muestra de 38 estudiantes seleccionados al azar para adecuar el instrumento. El instrumento presentó un alfa de Cronbach de 0.958, que representa buena consistencia interna. Por su parte, en los estudios realizados por Valero en los que se aplicó el CAEX se encontraron alfas de Cronbach de 0.857-0.923.

El cuestionario consta de 50 ítems, sus repuestas son del tipo Likert con puntuación ordinal del o al 5 para que el estudiante informase la frecuencia con que esas situaciones le ocurrían a él personalmente (o no siento nada, 1 ligera ansiedad, 2 un poco intranquilo, 3 bastante ansioso, 4 muy ansioso, 5 completamente ansioso). De igual forma, este instrumento mide las características comportamentales de la ansiedad de examen incluyendo: (1) las respuestas efectivas de evitación que se dan en los exámenes, (2) las respuestas cognitivas de preocupación ante o durante el examen, (3) las respuestas fisiológicas que efectivamente le ocurren al estudiante en ese momento y (4) las situaciones o tipos de exámenes más frecuentes.

Para efectos de clasificación de la ansiedad en niveles, se consideró la media y desviación estandar en cada una de las dimensiones y en el resultado total del CAEX. La ansiedad se dividió por rangos: Muy Baja (o-59 puntos), Baja (60-102 puntos), Media (103-146 puntos), Alta (147-189) y Superior (190-250 puntos).

La obtención del rendimiento académico de los estudiantes se obtuvo mediante la revisión del reporte digital de notas obtenidas en el OSCE. La clasificación de estos últimos datos se ajustó al sistema de notas definido en el reglamento del alumno regular de pregrado de la institución universitaria en la cual se desarrolló el estudio, obedeciendo a los siguientes valores: 6.1-7 sobresaliente; 5.1-6 muy bueno; 4.1-5 bueno; 3.1-4 suficiente; 2.1-3 menos que suficiente; 1.1-2 deficiente; 1 mínima.

Para la organización, procesamiento y análisis estadístico de la información se utilizó el software estadístico SPSS versión 21. Se utilizó, en primera instancia, la estadística descriptiva para cada una de las variables, considerando medidas de tendencia central y dispersión, posteriormente se avanzó con estadística inferencial mediante pruebas paramétricas como T-de Student y ANOVA.

Durante la investigación se respetaron los ocho requisitos éticos de Emanuel Ezequiel ${ }^{14}$, entre ellos se solicitó la autorización por parte del autor del instrumento CAEX. El proyecto fue evaluado por el Comité de Ética Científico de la Universidad Católica del Maule. Previo a la recolección de datos se solicitó permiso a las autoridades de la Escuela de Enfermería y el consentimiento informado 
a cada participante, se detalló el propósito del estudio, asegurándoles la confidencialidad de sus respuestas, así como el carácter voluntario de su participación. Todo el proceso se realizó bajo la supervisión del investigador responsable.

\section{RESULTADOS}

Las características de los estudiantes que participaron en este estudio se muestran en la Tabla 1. Como puede observarse, en mayor medida (46\%), correspondían al tercer semestre y una tercera parte al séptimo semestre (30\%). Como era lo esperado, la mayor parte eran adultos jóvenes, $75 \%$ tenía entre 21 y 25 años y 19\% entre 18 y 20 años; asimismo, en su mayoría (80\%) eran del sexo femenino. Poco más de la mitad procedían de la zona urbana (56\%), y el resto de la rural. También un mayor porcentaje procedía de un establecimiento particular subvencionado (55\%), en menor medida de un establecimiento municipal (39\%) y sólo un porcentaje mínimo de un establecimiento particular. Únicamente $17 \%$ de los estudiantes además de estudiar también trabaja.

El promedio total del rendimiento académico de este grupo fue de 5.1. En la Tabla 2 se presentan los promedios del rendimiento académico en relación a las características sociodemográficas.

En términos generales, el rendimiento de acuerdo a estas variables fue bueno y muy bueno, únicamente en la distinción según semestre los estudiantes del tercero tuvieron promedio menor a 5; en la distribución en los otros semestres y en las otras variables el promedio de rendimiento estuvo entre 5 y 5.4. El rendimiento académico en el OSCE, entre los distintos semestres, mostró que los estudiantes del tercer semestre presentaron un rendimiento que los ubica en rendimiento bueno, el cual es menor con relación a los semestres posteriores donde el promedio obtenido corresponde a muy bueno, esta diferencia fue estadísticamente significativa. Asimismo, en la relación entre rendimiento y ocupación se observó mayor rendimiento en los estudiantes que en paralelo trabajan y estudian respecto a los que sólo estudian, esta diferencia también alcanzó significancia estadística.

El promedio de ansiedad en este grupo estudiado fue de 103.5 puntos, lo que corresponde a un nivel de ansiedad media. En la Tabla 3 se muestra la relación entre el promedio de ansiedad en cada una de las variables sociodemográficas incluidas. En todas las variables el promedio del nivel de ansiedad obtenido corresponde a un nivel medio o bajo; los promedios menores se observaron entre los alumnos de mayor edad (26 a 30 años), los hombres, y quienes cursaban el séptimo semestre, es decir, éstos fueron quienes presentaron menor nivel de ansiedad. No obstante, únicamente en la distinción por sexo las diferencias fueron estadísticamente significativas, ya que el sexo femenino presentó mayor ansiedad ante el examen OSCE.

\begin{tabular}{lr}
\hline \multicolumn{2}{l}{$\begin{array}{l}\text { Tabla 1. Caracterización de la } \\
\text { muestra de estudio }(\mathbf{n}=94)\end{array}$} \\
\hline Variable & $\%$ \\
\hline Semestre Académico & \\
\hline Tercero & 46 \\
\hline Quinto & 25 \\
\hline Séptimo & 30 \\
\hline Edad & \\
\hline 18-20 & 19 \\
\hline $21-25$ & 75 \\
\hline $26-30$ & 5 \\
\hline 31 y+ & 1 \\
\hline Sexo & \\
\hline Femenino & 80 \\
\hline Masculino & 20 \\
\hline Procedencia & \\
\hline Rural & 44 \\
\hline Urbano & 56 \\
\hline Colegio de procedencia & \\
\hline Municipal & 39 \\
\hline Particular/subvencionado & 55 \\
\hline Particular & 5 \\
\hline Ocupación & \\
\hline Estudiante & 83 \\
\hline Estudiante/trabaja & 17 \\
\hline
\end{tabular}


Tabla 2. Rendimiento académico y su relación

con las variables sociodemográficas $(n=94)$

\begin{tabular}{|c|c|c|c|}
\hline Variables & Promedio de rendimiento & $\mathbf{F}$ & $\mathbf{P}$ \\
\hline \multicolumn{4}{|l|}{ Semestre Académico } \\
\hline Tercero & 4.9 & 4.4 & $* 0.015$ \\
\hline Quinto & 5.2 & & \\
\hline Séptimo & 5.3 & & \\
\hline \multicolumn{4}{|l|}{ Edad } \\
\hline $18-20$ & 5.1 & 0.72 & 0.53 \\
\hline $21-25$ & 5.1 & & \\
\hline $26-30$ & 5.1 & & \\
\hline \multicolumn{4}{|l|}{ Sexo } \\
\hline Femenino & 5.1 & 0.4 & 0.84 \\
\hline Masculino & 5.1 & & \\
\hline \multicolumn{4}{|l|}{ Procedencia } \\
\hline Rural & 5.0 & 2.5 & 0.64 \\
\hline Urbano & 5.2 & & \\
\hline \multicolumn{4}{|l|}{ Colegio de procedencia } \\
\hline Municipal & 5.2 & 1 & 0.37 \\
\hline Particular/subvencionado & 5.0 & & \\
\hline Particular & 5.0 & & \\
\hline \multicolumn{4}{|l|}{ Ocupación } \\
\hline Estudia & 5.1 & 5.05 & $* 0.027$ \\
\hline Estudia/trabaja & 5.4 & & \\
\hline
\end{tabular}

\section{DISCUSIÓN}

En este estudio, respecto a las variables centrales consideradas, el rendimiento académico correspondió en mayor medida a un nivel muy bueno y el nivel de ansiedad identificado se encontró entre los rangos medios y bajos. Se pudo observar que las manifestaciones de ansiedad ante exámenes no expresaron niveles elevados y no mostraron relación con el rendimiento académico. Este resultado es relevante ya que muestra un cambio generacional sobre cómo los estudiantes están enfrentando los exámenes, lo que pudiera atribuirse a que el proceso formativo en competencias ha organizado su malla curricular de manera efectiva. Dicho proceso entrega al estudiante experiencias previas con talleres, desarrollo de casos clínicos, evaluaciones formativas y prácticas clínicas que favorecen el proceso de enseñanza y aprendizaje y le permiten enfrentar en mejores condiciones los distintos tipos de exámenes universitarios. Aunado a lo anterior, en la formación de enfermería también se utiliza la simulación clínica, la cual ofrece una oportunidad única de aprendizaje y de participación, pues se fomenta el logro de altos niveles de rendimiento clínico ${ }^{15,16}$.

Por otra parte, estos resultados difieren de los observados en otros estudios en los cuales los alumnos manifestaron una alta ansiedad ante los exámenes y se mermó el desempeño académico ${ }^{17,18}$. En relación al rendimiento académico se observaron diferencias significativas con el 
Tabla 3. Ansiedad ante exámenes y su relación con las variables sociodemográficas $(n=94)$

\begin{tabular}{lccc}
\hline Variables & Promedio de ansiedad & F & P \\
Semestre Académico & 108.51 & 0.579 & 0.563 \\
\hline Tercero & 101.22 & & \\
\hline Quinto & 97.54 & & \\
\hline Séptimo & & & \\
Edad & 95.17 & 1.857 & 0.143 \\
\hline $18-20$ & 108.54 & & \\
\hline $21-25$ & 69.80 & & \\
\hline $26-30$ & & & \\
\hline Sexo & 108.12 & 1.788 & $* 0.038$ \\
\hline Femenino & 85.05 & & \\
\hline Masculino & & & \\
\hline Procedencia & 107.55 & 0.337 & 0.799 \\
\hline Rural & 109.33 & & \\
\hline Urbano & & & \\
\hline Colegio de procedencia & 98.16 & 0.725 & 0.487 \\
\hline Municipal & 105.58 & & \\
\hline Particular/subvencionado & 120.60 & & \\
\hline Particular & & & \\
\hline Ocupación & 101.65 & & \\
\hline Estudia & 112.25 & 0.791 & \\
\hline Estudia/trabaja & & & \\
\hline & & & \\
\hline
\end{tabular}

nivel que cursa el estudiante, por ejemplo, aquellos inscritos en cursos más avanzados presentaron un mejor desempeño académico. Este resultado se puede sustentar desde la Teoría de Benner, De principiante a Experto, donde se refiere que la adquisición de habilidades se desarrolla de acuerdo a niveles?

Según este modelo, los principiantes son aquellos que no han tenido experiencia en acciones que se espera realicen y se enfocan en seguir las reglas dictadas por su supervisor clínico. En este escenario se encontrarían los estudiantes de los primeros niveles académicos del presente estudio, quienes presentaron un mayor nivel de ansiedad y un menor desempeño académico. En los primeros años de formación los estudiantes comienzan a relacionar el conocimiento teórico con el práctico y se enfrentan por primera vez a situaciones que demandan una mayor responsabilidad, lo que genera nerviosismo y ansiedad. Por el contrario, en los últimos niveles de formación en enfermería se encuentran aquellos que Benner denomina como principiante avanzado, es decir, estudiantes que obtuvieron mejor rendimiento académico, ya que en esta etapa comienzan a dominar la práctica clínica, sintiéndose más seguros de las acciones que realizan, además de que usan la experiencia práctica para resolver situaciones y enfrentar de manera más eficiente los distintos escenarios clínicos. 
En ese sentido, la teoría de Benner fortalece la idea de que la experiencia clínica enriquece la formación del estudiante de enfermería, y pudiera tener relación con lo que se observó en este estudio, puesto que en etapas iniciales se presentan temores e inseguridades al enfrentarse por primera vez a situaciones complejas como lo es el examen OSCE. Sin embargo, a medida que el estudiante avanza a niveles superiores el aprendizaje se va logrando, van adquiriendo habilidades y destrezas que sólo son propias del quehacer clínico. Algunos estudios explican esta condición de aprendizaje con las situaciones altamente estresantes que enfrentarían los estudiantes de enfermería durante sus prácticas clínicas ${ }^{19-21}$ en etapas iniciales.

Otro aspecto significativo en este estudio es la relación entre el sexo del estudiante y la ansiedad. En este caso las mujeres presentan mayor ansiedad frente a exámenes, lo que se correlaciona con otras investigaciones que también muestran que las mujeres tienen mayores niveles de ansiedad que los hombres ante exámenes ${ }^{22-24}$. Este hecho, según algunos autores, se asociaría a la función del género ${ }^{25}$.

Con respecto a la variable ocupación del estudiante, la cual mostró diferencia significativa con el rendimiento académico, los resultados arrojaron que los estudiantes que trabajan y estudian de forma paralela presentan un mejor rendimiento académico y sus niveles de ansiedad son medios. Dicho resultado coincide con el estudio de Caballero, quien menciona que el trabajo puede tener efectos positivos ${ }^{25,26}$ en el desempeño académico, dado que favorece la transferencia de conocimientos relacionados con los temas de estudios, o si no está relacionado con la carrera, refuerza el sentido de responsabilidad y disciplina en el desempeño académico.

Si bien el estudiante trabajador se favorece del aprendizaje laboral para enfrentar el estrés académico, trabajar y estudiar tiene algunos efectos negativos. De acuerdo con Barreto et al., los alumnos reconocen ciertas desventajas al realizar ambas actividades en paralelo como: contar con menos tiempo para estudiar, tener poco tiempo libre y escasa participación de las actividades académicas, en comparación con los que sólo se dedican a estudiar ${ }^{27,28}$. Ahora bien, en el presente estudio el resultado se puede entender ya que los estudiantes que trabajan van adquiriendo habilidades y competencias generales con el paso del tiempo, las cuales los preparan de mejor manera para enfrentarse al mundo académico.

Aunque en esta investigación los niveles de ansiedad no son elevados, la presencia de ansiedad frente a exámenes, cualquiera que sea su prevalencia, es una condición de salud mental que debe ser atendida en el contexto académico debido a las fuertes implicaciones que la misma puede tener sobre el desempeño del estudiante ${ }^{6}$. Asimismo, Rodríguez et al. afirman que experimentar ansiedad ante los exámenes, por baja que sea, es lo suficientemente fuerte como para considerarse un problema y, por lo tanto, es imprescindible buscar una solución ${ }^{21}$. Esto último pone de manifiesto la necesidad de desarrollar estrategias educativas, identificar tempranamente estudiantes de mayor riesgo e iniciar intervenciones integrales en salud de manera oportuna y eficaz.

\section{CONCLUSIÓN}

En relación al estudio, cabe destacar que el instrumento CAEX se aplicó por primera vez en población chilena. Si bien los niveles de ansiedad en dicha población se concentraron en niveles bajos y medios, la ansiedad es un fenómeno que requiere de una prevención precoz por las posibles consecuencias negativas que puede provocar en la salud mental de los estudiantes. 
Se puede concluir que 58.3\% de los estudiantes con rendimiento en el OSCE fue bueno y muy bueno, presentando un nivel de ansiedad de media a baja, también se observaron diferencias significativas entre las variables sexo y el nivel de ansiedad, siendo las mujeres las que presentan mayor ansiedad ante el examen del OSCE. Igualmente, existe relación significativa entre el rendimiento y la ocupación del estudiante que trabaja y estudia, presentando mayor rendimiento los estudiantes que realizan en paralelo ambas actividades.

Dentro de los resultados relevantes se destaca esto último, el mejor desempeño académico del estudiante trabajador frente al que sólo se dedica a estudiar. La información obtenida genera un desafío importante para las escuelas de enfermería, en el sentido de reestructurar sus mallas curriculares para flexibilizar los horarios académicos y permitir el desarrollo de ambas actividades, trabajo y estudio, de manera que se pueda disminuir el agobio y sobrecarga académica. Más aún, resulta imprescindible incluir programas de promoción de la salud mental en los jóvenes universitarios que respondan a las necesidades específicas de este grupo, adecuadas al contexto socio-político-económico-educacional de cada casa de estudio, procurando mejorar la calidad de vida de los estudiantes.

Se puede considerar una limitación del estudio el hecho de tener sólo una muestra local de estudiantes de enfermería, por lo tanto, sería importante aplicar en futuras investigaciones el instrumento CAEX con otras poblaciones de estudiantes de enfermería, así como en otros estudiantes de carreras de ciencias de la salud.

De igual forma, se recomienda analizar otras variables que puedan afectar directamente los síntomas físicos, psicológicos y comportamentales manifestados por la ansiedad académica. Finalmente, se debe realizar estudios que permitan relacionar las variables aquí estudiadas con otras, como horas dedicadas al estudio, principales estresores académicos, herramientas de aprendizaje utilizadas por los estudiantes y medidas de afrontamiento frente a situaciones estresantes.

\section{RESPONSABILIDADES ÉTICAS}

Protección de personas y animales. En esta investigación no se realizaron experimentos con personas o animales. La investigación se ajustó a los principios éticos vigentes en la institución en la que se realizó el estudio.

Confidencialidad. En esta investigación no aparecen datos personales que permitan identificar a los participantes, a quienes se les garantizó el anonimato y la confidencialidad de la información.

Conflicto de intereses. Los autores declaran no tener ningún conflicto de intereses.

Financiamiento. No se recibió patrocinio para llevar a cabo este estudio.

\section{REFERENCIAS}

1. Alarcón A. Incorporación del Examen Clínico Objetivo Estructurado (ECOE) en la carrera de enfermería. Rev. educ. ciencias salud. 2013; 10(1): 18-22. http://bit.ly/396jKwq

2. Barrios-Araya S, Masalán-Apip MP, Cook MP. Educación en salud: en la búsqueda de metodologías innovadoras. Cienc. enferm. 2011; 17(1): 57-69.

http://dx.doi.org/10.4067/S0717-95532011000100007

3. Brykczynski K. Patricia Benner: Cuidado, sabiduría clínica y ética en la práctica de enfermería. En: Marriner-Tomey A. Modelos y teorías de enfermería. $8^{\text {va }}$ ed. España: Elsevier; 2014. 
4. Triviño X, Vasquez A, Mena A, López A, Aldunate M, Varas M, et al. Aplicación del Examen Clínico Objetivo Estructurado (OSCE) en la evaluación final del internado de pediatría en dos escuelas de medicina. Rev. méd. Chile. 2002; 130(7): 817-24.

http://dx.doi.org/10.4067/S0034-98872002000700016

5. Gutiérrez-Calvo M, Avero P. Ansiedad, estrategias auxiliares y comprensión lectora: déficit de procesamiento versus falta de confianza. Psicothema. 1995; 7(3): 569-78. https://bit.ly/2OUjUAo

6. Ávila-Toscano JH, Hoyos-Pacheco SL, González DP, y Cabrales-Polo A. Relación entre ansiedad ante los exámenes, tipos de pruebas y rendimiento académico en estudiantes universitarios. Psicogente. 2011; 14(26): 255-68. http://bit.ly/3r7OoE1

7. Fernández A. Cambios contextuales y su efecto sobre las puntuaciones en los exámenes. Psicológica. 1990; 11: 1-8. https://bit.ly/312fQ_-L

8. González-Olaya HL, Delgado-Rico HD, Escobar-Sánchez M, Cárdenas-Angelone ME. Asociación entre el estrés, el riesgo de depresión y el rendimiento académico en estudiantes de los primeros semestres de un programa colombiano de medicina. FEM. 2014; 17(1): 47-54.

http://dx.doi.org/10.4321/S2014-98322014000100008

9. Marty M, Lavín M, Figueroa M, Larraín D, Cruz C. Prevalencia de estrés en estudiantes del área de la salud de Universidad de los Andes y su relación con enfermedades infecciosas. Rev. chil. neuropsiquiatr. 2005; 43(1): 25-32. http://dx.doi.org/10.4067/So717-92272005000100004

10. Pradhan G, Mendinca NL, Kar M. Evaluation of examination stress and its effec on cognitive function among first year medical studens. J Clin Diagn Res. 2014; 8(8): BC05-7.

https://doi.org/10.7860/JCDR/2014/9014.4680

11. Castillo-Pimienta C, Chacón-de la Cruz T, Díaz-Véliz G. Ansiedad y fuentes de estrés académico en estudiantes de carreras de la salud. Investigación educ. médica. 2016; 5(20): 230-7.

https://doi.org/10.1016/j.riem.2016.03.001

12. Pozos-Radillo BE, Preciado-Serrano ML, Plascencia-Campos A, Acosta-Fernández M, Aguilera MA. Estrés académico y síntomas físicos, psicológicos y comportamentales en estudiantes mexicanos de una universidad pública. Ansiedad estrés. 2015; 21(1): 35-42. http://bit.ly/3lCOF8x

13. Valero-Aguayo L. Evaluación de ansiedad ante exámenes: datos de aplicación y fiabilidad de un cuestionario CAEX. An. psicol. 1999;15(2): 223-31. https://bit.ly/3tPxsvD

14. Suárez-Obando F. Un marco ético amplio para la investigación científica en seres humanos: más allá de los códigos y las declaraciones: la propuesta de Ezekiel J. Emanuel. pers. bioét. 2015; 19(2): 182-97. https://doi.org/10.5294/PEBI.2015.19.2.2

15. Seibert DC, Guthrie JT, Adamo G. Improving learning outcomes: integration of standardized patients \& telemedicine technology. Nurs Educ Perspect. 2004; 25(5): 232-7. http://bit.ly/2NGGA69

16. Aguilar-Rivera MC, Gil-Llanes OF, Pinto-Garrido VA, Quijada-Maldonado CR, Zúñiga-Sánchez CA. Inteligencia emocional, estrés, autoeficacia, locus de control y rendimiento académico en universitarios. Enseñ. invest. psicol. 2014; 19(1): 21-35. http://bit.ly/3r5yG3W

17. Barrios-Araya S, Urrutia-Egaña M, Rubio-Acuña M. Impacto de la simulación en el desarrollo de la autoeficacia y del locus de control en estudiantes de enfermería. Educ Med Super. 2017. 31(1): 125-36. https://bit.ly/3sg8ipA

18. Álvarez J, Aguilar JM, Lorenzo JJ. La ansiedad ante los exámenes en estudiantes universitarios: relaciones con variables personales y académicas. Rev Electron Investig Psicoeduc Psigopedag. 2012; 10(1): 333-54. http://bit.ly/3vP9rgF 
19. Barraza R, Muñoz N, Alfaro M, Álvarez A, Araya V, Villagra C, et al. Ansiedad, depresión, estrés y organización de la personalidad en estudiantes novatos de medicina y enfermería. Rev. chil. neuropsiquiatr. 2015; 53(4): 251-60. http://dx.doi.org/10.4067/So717-92272015000400005

20. De Dios-Duarte MJ, Varela-Montero I, Braschi-Diaferia L, Sánchez-Muñoz E. Estrés en estudiantes de enfermería. Educ Med Super. 2017; 31(3): 110-23. https://bit.ly/3scWp3n

21. Rodríguez A, Dapía MD, López-Castedo A. Ansiedad ante los exámenes en alumnado de educación secundaria obligatoria. R Est Inv Psico Educ. 2014; 1(2): 132-40. https://ng.cl/cpybc

22. Tessa R. Factores estresores percibidos por los estudiantes de la facultad de enfermería de la Universidad Católica de Uruguay en la práctica clínica. Rev. Colomb. Enferm. 2015. 10; 27-34. https://doi.org/10.18270/rce.v10i10.577

23. Casari LM, Anglada J, Daher C. Estrategias de afrontamiento y ansiedad ante exámenes en estudiantes universitarios. Revista de Psicología. 2014; 32(2): 243-69. http://bit.ly/316UEJt

24. Herrera LM, Rivera MS. Prevalencia de malestar psicológico en estudiantes de enfermería relacionada con factores sociodemográficos, académicos y familiares. Cienc. enferm. 2011; 17(2): 55-64. http://dx.doi.org/10.4067/SO717-95532011000200007

25. Caballero CC, Abello R, Palacio J. Relación del burnout y el rendimiento académico con la satisfacción frente a los estudios en estudiantes universitarios. Av. Psicol. Latinoam. 2007; 25(2): 98-111. http://bit.ly/3rlvNfH

26. García-Rodríguez JJ, Labajos-Manzanares MT, Fernández-Luque F, Características personales de los estudiantes de enfermería que les hacen vulnerables al estrés. Revista Enfermería Docente. 2014; (102): 20-6.

27. Cruz-Ponce R, González-Franco D. Rendimiento académico entre alumnos del tercer año de la carrera de Médico Cirujano que trabajan y que no trabajan. Rev. Esp. Med-Ouir. http://bit.ly/2OrRKg5

28. Barreto-Osma DA, Celis-Estupiñan CG, Pinzón-Arteaga IA. Estudiantes universitarios que trabajan: subjetividad, construcción de sentido e insatisfacción. Rev. virtual. univ. catol. norte. 2019; (58); 96-115. https://doi.org/10.35575/rvucn.n58a4 